\title{
Automatic identification and counting of small size pests in greenhouse conditions with low computational cost
}

\author{
Chunlei Xia ${ }^{\mathrm{a}, \mathrm{b}}$, Tae-Soo Chon ${ }^{\mathrm{c}}$, Zongming Ren ${ }^{\mathrm{d}}$, Jang-Myung Lee ${ }^{\mathrm{b}, *}$ \\ a The Research Center for Coastal Environmental Engineering and Technology of Shandong Province, Yantai Institute of Coastal Zone Research, Chinese Academy of Sciences, Yantai 264003, \\ P.R. China \\ b School of Electrical Engineering, Pusan National University, Busan (Pusan) 609-735, Republic of Korea \\ c Department of Biological Sciences, Pusan National University, Busan (Pusan) 609-735, Republic of Korea \\ ${ }^{d}$ College of Life Science, Shandong Normal University, Jinan, 250014, P. R. China
}

\section{A R T I C L E I N F O}

\section{Article history:}

Received 31 March 2014

Received in revised form 12 September 2014

Accepted 15 September 2014

Available online 28 September 2014

\section{Keywords:}

Pest monitoring

Computational complexity

Mahalanobis distance

Greenhouse management

\begin{abstract}
A B S T R A C T
We propose an automatic pest identification method suitable for large scale, long term monitoring for mobile or embedded devices in situ with less computational cost. A procedure of segmentation and image separation was devised to identify common greenhouse pests, whiteflies, aphid and thrips. Initially, the watershed algorithm was used to segment insects from the background (i.e., sticky trap) images. Color feature of the insects were subsequently extracted by Mahalanobis distance for identification of pest species. Accuracy and computational costs were evaluated across different image resolutions. The correlation of determination $\left(R^{2}\right)$ between the proposed identification scheme and manual identification were high, showing 0.934 for whitefly, 0.925 for thrips, and 0.945 for aphids even with low resolution images. Comparing with the conventional methods, pests were efficiently identified with low computational cost. Optimal image resolution for species identification regarding long-term survey was discussed in practical aspect with less computational complexity.
\end{abstract}

(c) 2014 Elsevier B.V. All rights reserved.

\section{Introduction}

Pest is one of the critical factors causing economic loss in greenhouse where crops are cultivated in congested conditions in limited areas. Integrated Pest Management (IPM) has been widely applied to the agricultural practices to achieve minimizing crop damage, environmental contamination, and economic loss concurrently (Allen and Rajotte, 1990). One of prerequisites for IPM, however, is to accurately investigate population densities of pest species. Objective identification of pest species and density estimation is essential for initiating any pest management program (Qiao et al., 2008). One of the most common methods for pest detection in greenhouse, however, has been mainly based on conventional sticky traps (Pinto-Zevallos and Vänninen, 2013). Counting the number of insects on sticky traps has been conventionally relied on visual judgment by humans (Wise et al., 2007). Due to complexity of insect morphology automatic identification has been considered as a difficult task. Especially with the small size pests, such as greenhouse insect pests, the efficiency of human counting is low and unreliable depending on observation conditions of observers (e.g., identification skill, fatigue). Therefore, implementation of automatic pest identification is vital to the modern agricultural production. Since the automatic pest identification system is mainly based on the

\footnotetext{
* Corresponding author. Tel.: + 82515102378

E-mail address: jmlee@pusan.ac.kr (J.-M. Lee).
}

visual information (e.g., pest shape, color), it would not produce any extra disturbances to environment including chemical/physical pollutions and could not raise degradation issues to the ecosystems. The ecological intensification could be achieved by employing the automatic pest identification in the agricultural practices that maximizes the production while minimizing anthropogenic environmental impacts concurrently (Bommarco et al., 2013).

Since the last decade computer hardware and imaging devices significantly contributed to automatic identification of biological organisms (Gaston and O'Neill, 2004; MacLeod et al., 2010). Detection of agricultural pests has garnered special attention, especially greenhouse pests such as whitefly (Bemisia tabaci Genn), aphids (Aphis gossypii Glover) and thrips (Thrips tabaci L.). These greenhouse pests are small in size and difficult to recognize, but are critical in causing damage under congested cultivation conditions. Martin and Thonnat (2007) presented a cognitive vision approach that adjusts optimal parameters for segmenting whitefly out of leaves based on adaptive learning techniques. By employing machine vision and knowledge-base techniques, Boissard et al. (2008) proposed a multidisciplinary cognitive vision approach applicable to whitefly detection in rose in situ. Solis Sánchez et al. (2009) utilized the geometric features (e.g., eccentricity, area size) to whitefly scouting by segmenting the insects from sticky trap images. Xia et al. (2012) developed a multifractal dimension to detect whitefly in situ, which was robust to field noise such as unequal illumination changes and light reflections on trap surface. 
Previous automatic identification, however, was mainly conducted with one species, whiteflies. Other common species such as aphids and thrips are also important in greenhouse conditions but were not extensively studied with image processing. Cho et al. (2007) proposed an automatic pest identification method based on YUV color space and fixed thresholds to examine pests from the sticky traps collected in greenhouse. Color components and body size of pest were utilized to identify whitefly, aphids and thrips in this study, but identification had to be conducted with high resolution images (600 DPI) only. Gabor filter and histogram of gradients were additionally utilized to extract the feature of pests in order to identify whitefly and greenfly from sticky trap in field conditions by Kumar et al. (2010), and they applied the support vector machine (SVM) to identification of the pests. SolisSánchez et al. (2011) utilized scale invariant feature transform (SIFT) as a feature descriptor of the segmented insect images, combining with shape feature of insect body. These methods, however, in general also require either high resolution of images (Cho et al., 2007; Solis-Sánchez et al., 2011) or computationally expensive recognition algorithms (e.g., Gabor filter, SIFT). Although high pest recognition accuracy was achieved by these methods, the methods are in practical aspect not specifically suitable for large scale, long term pest monitoring (e.g., agricultural sensing network) in field conditions due to high computational cost. Since portable devices or wireless sensor network are usually powered by small capacity batteries, the high computational cost would be prone to a high risk of power loss for data processing. An alternative solution may be to transmit the field images to the remote terminal (e.g., personal computer) for pest identification. But the transmission ability of the wireless network would be still a constraining factor since high resolution images would require a broadband network of which the transmission range is limited, especially in the large scale agricultural field (Koumpouros et al., 2004). Although recent 3G/4G mobile network may provide a technical solution to the transmission of images in large scale (i.e., data transmission between cites), the cost of the mobile network would not be affordable for agricultural practices by dealing with high resolution data. Effective pest recognition algorithm with low computation cost is desired for large scale pest monitoring in practical aspect.

In this paper, we propose a procedure of image processing suitable for species identification with low resolution images of small size insects by combining methods for image segmentation and separation. A classical segmentation algorithm, marker controlled watershed, was initially applied to the $\mathrm{YC}_{\mathrm{b}} \mathrm{C}_{\mathrm{r}}$ color space of sticky trap image. Subsequently Mahalanobis distance was used as the classification criterion for species identification by differentiating the color components and insect size. With less computational complexity based on these algorithms, efficient identification was achieved with reduction in computation time, while still maintaining high precision rate for identification of three major pests in greenhouse.

\section{Materials and methods}

Three species in greenhouse, whitefly (Bemisia tabaci Genn), aphids (Aphis gossypii Glover) and thrips (Thrips tabaci L.), which reported to cause serious damage in greenhouse crops over the world (Malais and Ravensberg, 2004), were selected as the target species for identification in this study. The sticky traps $(15 \mathrm{~cm} \times 10 \mathrm{~cm})$ were placed $10 \mathrm{~cm}$ above tomato crops that were approximately $160 \mathrm{~cm}$ tall. The borders of the sticky trap were encapsulated by cardboard with $2 \mathrm{~mm}$ thickness. In total, fifteen sticky traps were selected for collecting species for one week. Methods for plant cultivation and insect observation were reported in (Chung et al., 2014; Qiao et al., 2008). After field collection, sticky trap images were acquired by the HP G3110 ${ }^{\circledR}$ scanner. Ten of the sticky traps were randomly selected for developing the species identification program. The total number of pests attached on the ten sticky traps was ranged between 62 and 252 individuals per trap with the average number of pests of $115.2 \pm 53.9$ individuals per trap. The number of insects ranged in 13-152 individuals, 13-20 individuals and 27-83 individuals for whitefly, aphids, and thrips, respectively. Specifically the average was $42.0 \pm 39.7$ individuals per trap for whitefly, $17.2 \pm 8.8$ individuals per trap for aphids, and $59.8 \pm 20.4$ individuals per trap for thrips. To build the classifier for identification of three pest species, twenty samples for each pest species were randomly selected from the rest of 5 sticky traps.

For segmentation watershed was selected since the algorithm has been widely used in image processing including object detection (Boissard et al., 2008) and insect (i.e., whiteflies) body segmentation from field images (Xia et al., 2012). Direct application of the watershed segmentation, however, produces a number of unexpected regions in addition to the segmentation targets ( $\mathrm{Ng}$ et al., 2006). In order to solve this problem, the marker controlled watershed has been introduced as well to decrease the sensitivity to noise and to enhance the reliability of watershed segmentation (Parvati et al., 2008).

Mahalanobis distance has been reported to be effective in measuring the similarity of a set of given values to a set of values obtained from the sampled data. Mahalanobis distance was successful in image recognition or segmentation (e.g., character recognition, plant segmentation, plant disease identification) (Kato et al., 1999; Manh et al., 2001; Weinberger et al., 2006; Xia et al., 2013). In order to measure the similarity between given specimens to the three species, whitefly, aphids and thrips, variation of the body color was obtained from the insect samples. The Mahalanobis distance is defined as (De Maesschalck et al., 2000):

$D=\sqrt{(x-\mu)^{T} S^{-1}(x-\mu)}$

where $\mu$ is the mean color of the insects, $S^{-1}$ represents the inverse of covariance matrix $(S)$ of the color variations of the sampled insects, $T$ indicates the transpose operation, and $x$ is the input feature vector of given specimen to be classified. Mean colors and covariance matrices of each specimen image were obtained from the insect samples.

Species recognition was conducted by images across different resolutions. Different level of resolution was controlled by adjusting pixel numbers. The original image resolution acquired from the scanner was $3500 \times 2350$ pixels $(600 \mathrm{DPI})$, and converted to lower resolutions of $1600 \times 1100,1024 \times 690,800 \times 540$, and $640 \times 430$ pixels. The pest identification program was developed in Matlab 2011b (Mathworks) on a personal computer equipped with Intel G860® CPU with 4GB RAM memory.

\section{Procedure}

\subsection{Overall process}

The overall process of pest identification is presented in Fig. 1. First, individual insect images were segmented from the sticky trap on the $\mathrm{YC}_{\mathrm{b}} \mathrm{C}_{\mathrm{r}}$ color spaces. The marker controlled watershed segmentation was utilized to extract each individual insect from $C_{b}$ component of $\mathrm{YC}_{\mathrm{b}} \mathrm{C}_{\mathrm{r}}$ color spaces. Candidate insect images were determined by examining the area size of the segmented blobs. Each individual insect was represented by mean color of the insect body in HSV color space. Mahalanobis distance was then introduced as a classifier for identifying species.

Mean colors and covariance matrices of each specimen image were obtained from the sample specimen. In the identification process, Mahalanobis distances between the given image of insect and target species were calculated based on the obtained mean colors and covariance matrices. The specimen was identified to the species showing the nearest Mahalanobis distance. If the nearest Mahalanobis distance was longer than the predefined threshold which had been estimated from pretests, the candidate image was regarded as noise and was removed from the identification list. 


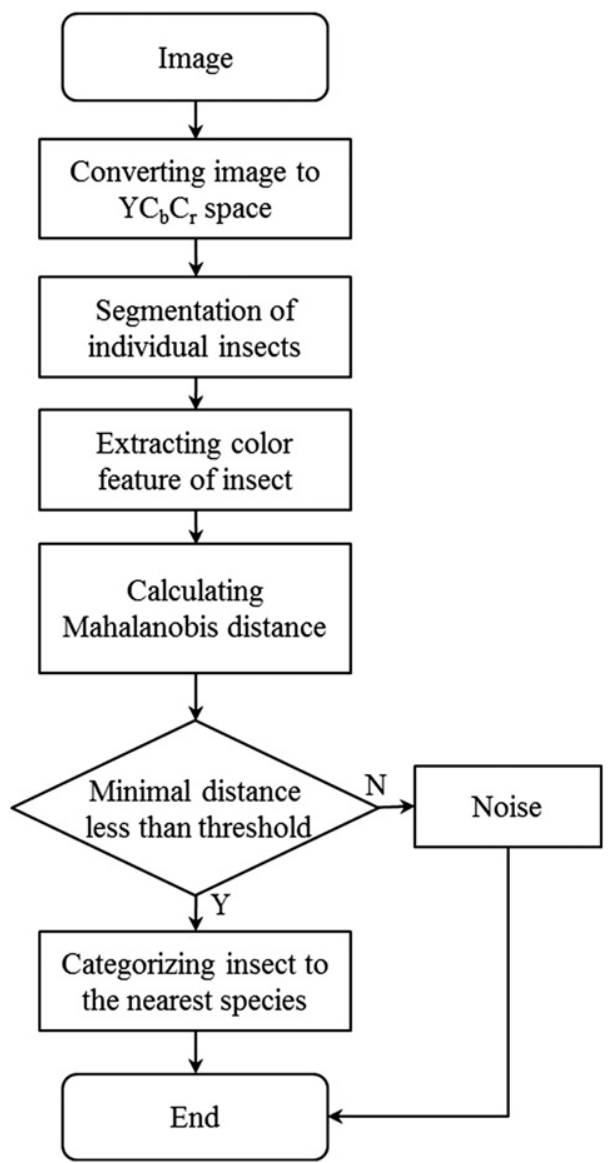

Fig. 1. Schematic flow of insect segmentation and species identification.

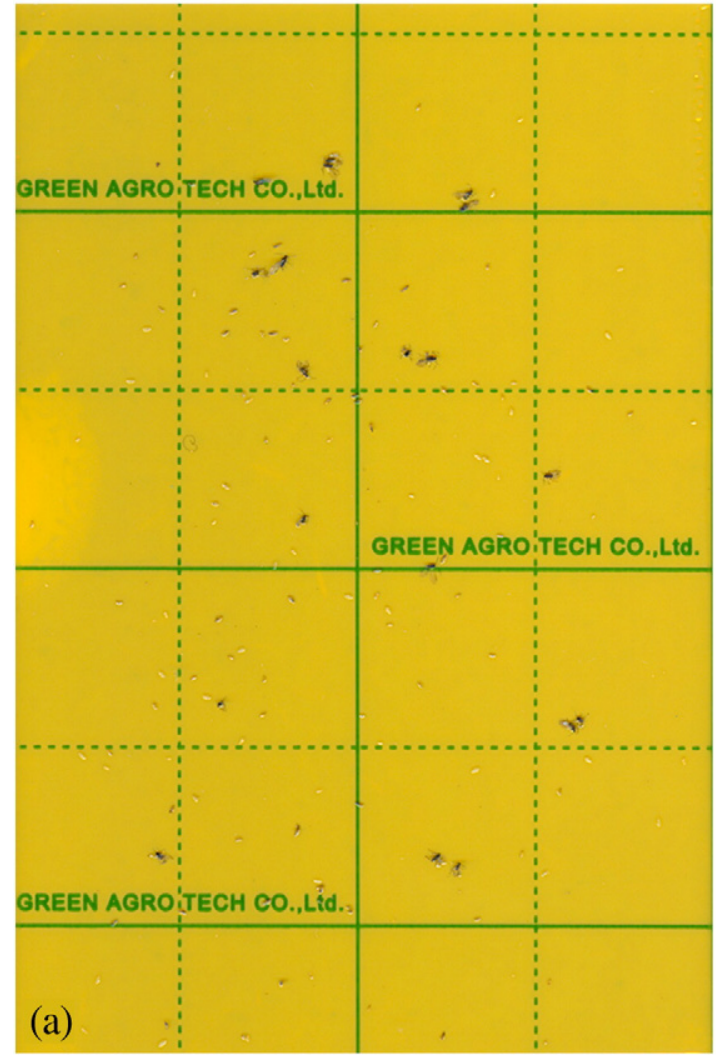

\subsection{Insect segmentation}

The sticky trap images mainly contain 5 colors of objects including yellow background, green grids, and characteristic tones of black (Aphids), white (Whitefly), and yellowish brown (Thrips) (Fig. 2a and b). Green color grids (and alphabets) printed on the yellow sticky trap was a strong source of heterogeneity against the yellow background of the sticky trap in the image. Indeed green grids caused severe noises in insect segmentation (Fig. 2a and c). Since the color of yellow, white and green has similar RGB values, it was difficult to accurately separate images of three species (Cho et al., 2007). In order to effectively distinguish the insects from grids and background, the sticky trap images need to be converted from RGB to other color spaces. In this study, three species were segmented in $\mathrm{YC}_{\mathrm{b}} \mathrm{C}_{\mathrm{r}}$ color space. Yellow background and green grids can be clearly distinguished from insects based on the $C_{b}$ component of $\mathrm{YC}_{\mathrm{b}} \mathrm{C}_{\mathrm{r}}$ color space. As shown in Fig. 2(c), intensity values of insects on $C_{b}$ component matched well to positions of individuals (Fig. 2a) and were much higher than the values of background and green grids. Consequently, the green grids, regarded as noise in this study, can be effectively removed from the sticky trap images.

The markers for conducting the watershed segmentation were obtained by calculating the regional minima and maxima. Watershed transform was then performed based on the regional maxima. Fig. 3(a) shows the segmented candidates of insects (Fig. 2a and c) with white blobs. Inaccurate segments, however, are also included within the insect candidates. After segmentation, the candidate images were determined by examining the area size of the segmented blobs produced by the watershed. The segmented blobs with improper size were further eliminated; green letters on the sticky trap (Fig. 2a) or local color distortion on the background of sticky trap usually produced large sized blobs.

According to the image resolution, appropriate range of body size was provided as a threshold to remove the unexpected large or small segments. For example, body area of insect was ranged in 2 to 60 pixels

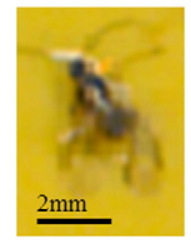

(b)
Aphids

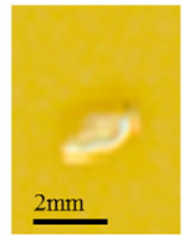

Whitefly

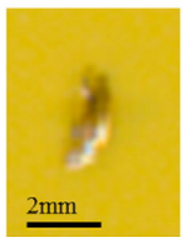

Thrips

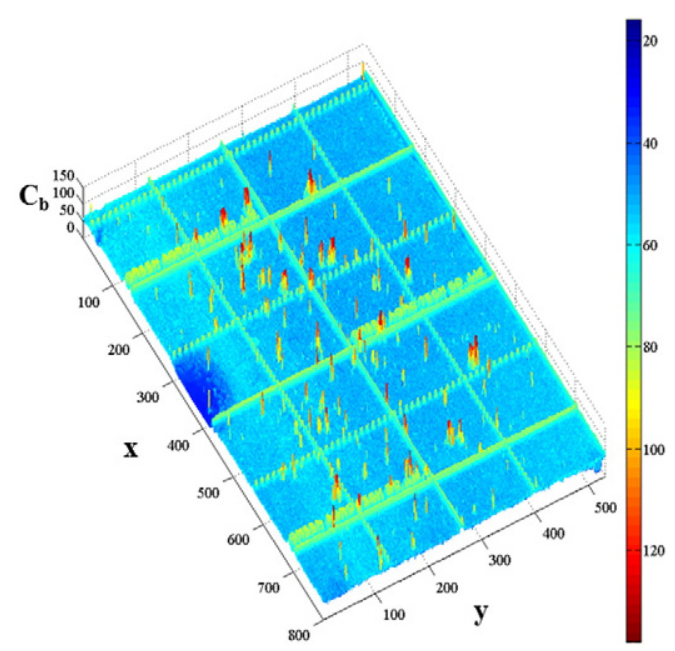

(c)

Fig. 2. Images of sticky trap and samples. (a) Trap, (b) three insect image recorded on the trap, and (c) $C_{b}$ component of sticky trap image. 


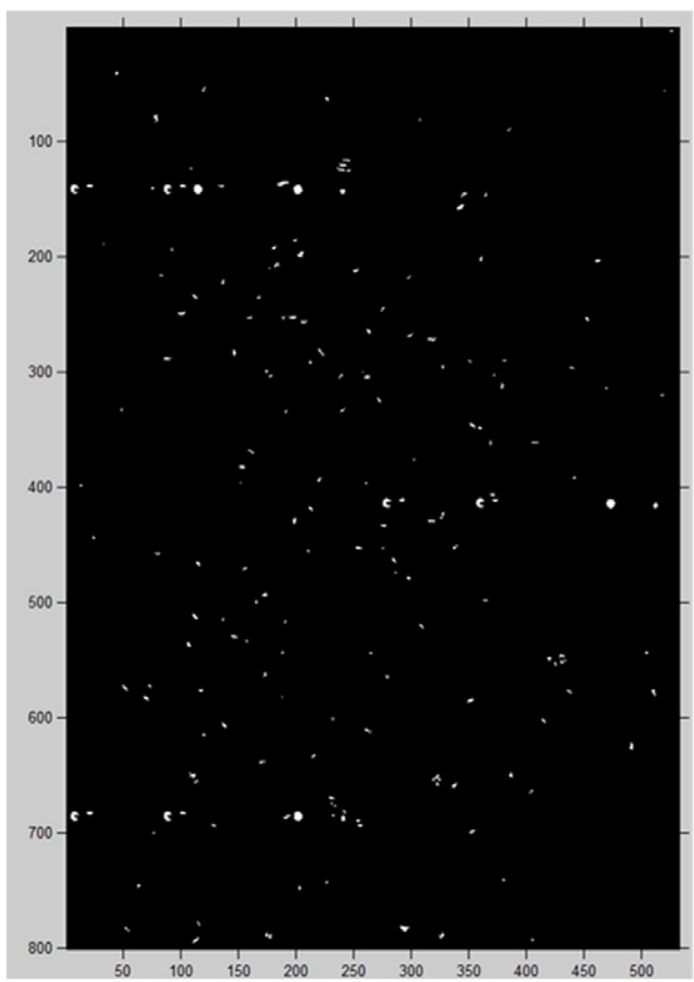

(a)

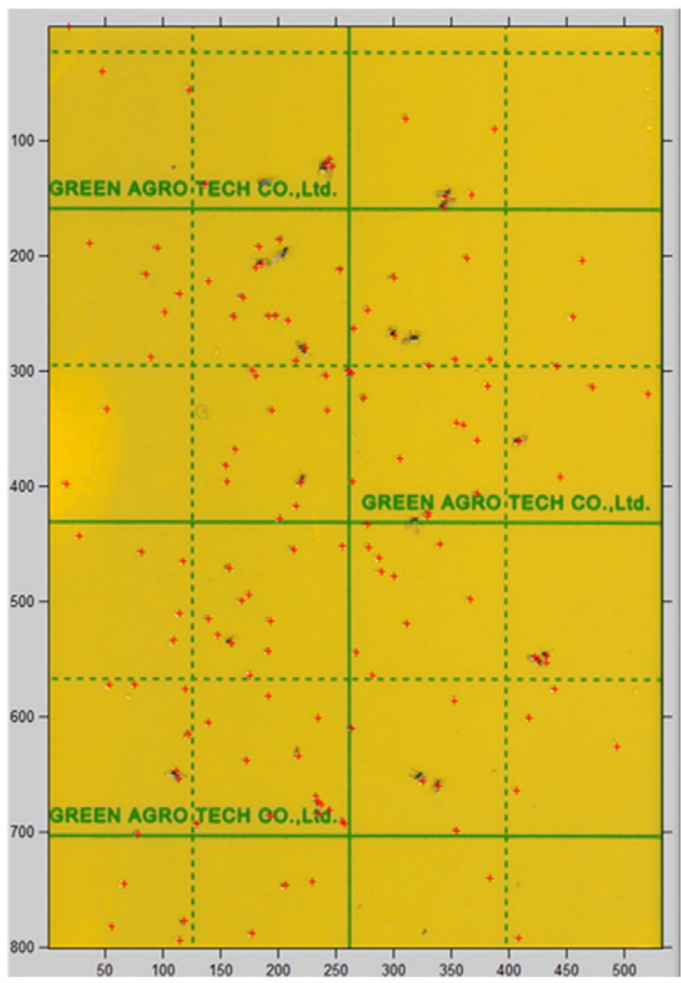

(b)

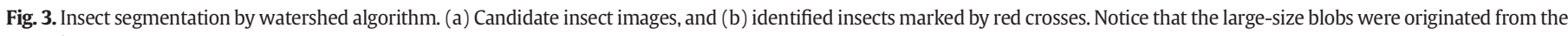
green letters.

with image resolution of $800 \times 540$ pixels. The ranges of insect area were mostly determined according to pretests across different resolutions. The determined insects were marked with red crosses as shown in Fig. 3. The unexpected large blobs were successfully removed from the segmentation results.

\subsection{Species identification}

After segmentation, images of individual insects are separated and extracted from the background (Fig. 4). The segmented blobs were numbered as shown in the left part of Fig. 4. The images of individuals were accordingly extracted and presented in the right panel of Fig. 4. Low image resolution $(800 \times 540$ pixels $)$ was presented in this case: the insect body size was less than 20 pixels. Therefore, the contours of the insects were not smooth and shapes of the insect could not be

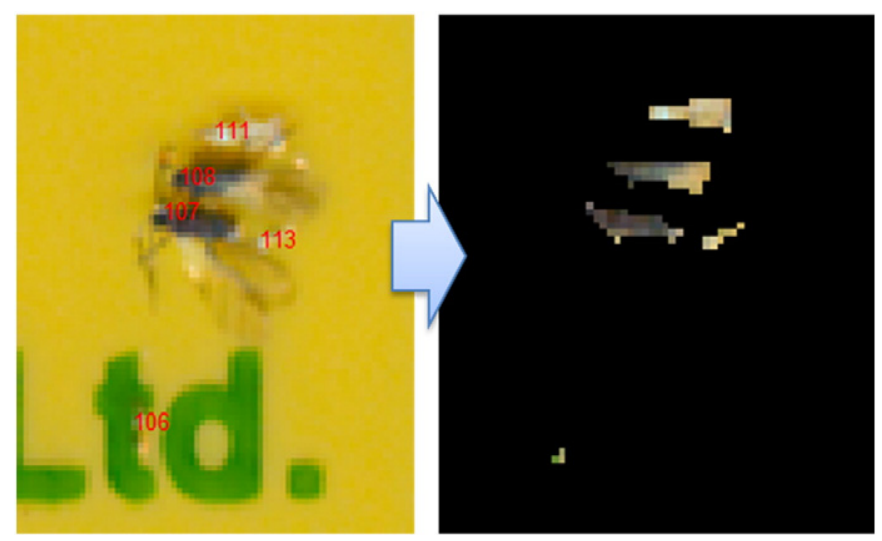

Fig. 4. Extraction of individual insects (Numbers indicating the sequence of segmented blobs). accurately identified based on size. However, three species, whitefly, aphids and thrips, showed different colors (Fig. 2b); the color features of these insects was in fact a critical factor for identifying pest species in this study.

The mean color $\mu$ and covariance matrix $S$ for Mahalanobis distance could be obtained from insect samples in different species. For the segmented insects (Fig. 4), mean color of the insect body was calculated as the input vector $x$. Three Mahalanobis distances were obtained for the input vector $x$ (of input specimen) to each of three species. By comparing the measured distances, the insect was classified to species showing the nearest distance. In this study, color vector $x$ was represented by HSV color instead of RGB and $\mathrm{YC}_{\mathrm{b}} \mathrm{C}_{\mathrm{r}}$. Although the insects were accurately segmented in the $\mathrm{YC}_{\mathrm{b}} \mathrm{C}_{\mathrm{r}}$ color space, the intensity value of these insect on $\mathrm{C}_{\mathrm{b}}$ component were similar and difficult to distinguish three species from these images. HSV color representation, however, demonstrated high performance in pest identification in the pre-tests. In addition, a predefined threshold value was given to examine the validation of the classification results. Species identification would be invalid if the nearest Mahalanobis distance is longer than the threshold. Since incorrectly-segmented blobs could be produced during the course of the segmentation process and could cause additional false alarms, noises were further removed by thresholding. The threshold was estimated from the preliminary tests; the value of 80 was used for all image resolutions.

\section{Experimental results}

Pest identification experiments were conducted in two steps: segmentation of individual insects from the sticky trap images and species identification of candidate images as stated above. Initially, detection of insect images was carried out by using the marker controlled watershed algorithm (see Section 3.2). The accuracy of insect segmentation and computational time were evaluated across different levels of resolution. 
The precision and recall analysis were applied to determining accuracy of insect detection (Xia et al., 2012). Precision is defined as the proportion of correct segmentation of insect (true positives) to the total segmentation results and is expressed as True positive / (True positive + False positive). Recall is measured as True positive / (True positive + False negative). The false positive could be also referred as false alarm since the non-insect (e.g., noise) is reported as an insect. In addition the false negative would stand for miss-detection of insects. The $F$ value based on precision and recall analysis represents the overall performance of the insect detection including miss detection and false alarms:

$F=\frac{2 \cdot \text { Precision } \cdot \text { Recall }}{\text { Precision }+ \text { Recall }}$.

High $F$ value indicates more correct detection with fewer false alarms at the same time. As presented in Fig. 5, the $F$ value varied from 0.96 to 0.92 ; overall performance across different image resolutions was in general over 0.90 . The highest $F$ value, 0.96 , was obtained from the 800 -pixel resolution, followed by 0.95 with the 640 -pixel resolution. The detection accuracy on 640-pixel resolution was only slightly lower than the best detection accuracy (800-pixel resolution). On the contrary, the detection accuracy tended to decrease as the image resolution increased higher than 800-pixel although the degree was slight. The images with the highest resolution that provided detailed information of insects showed lower detection accuracy $(F)$ in fact, although requiring much higher computational cost (Fig. 5). Due to oversegmentation in the higher resolution images, false alarms occurred frequently. Especially, the slight color changes on the yellow background could be easily confused with whitefly. The standard deviation was also presented as error bars with precision $(F)$ in Fig. 5. The highest standard deviation was found at the highest image resolution which indicated the segmentation performance with resolution of 3500-pixel was less robust to noises.

The computational time increased greatly with the increase in image resolution (Fig. 5). Especially, the image processing time with the highest resolution (3500-pixel, $600 \mathrm{DPI}$ ) increased 4 times ( $32.3 \mathrm{~s}$ ) comparing with the processing time with the 1600 -pixel resolution $(6.36 \mathrm{~s})$. This was 37 times longer than the processing time by the 640-pixel resolution $(0.87 \mathrm{~s})$. Since the computational time showed very large differences from the low to the high image resolution, the standard deviation was too small to plot in the line chart (Fig. 5). Instead, the standard deviation is separately provided in Fig. 6(a), showing higher values with increase in image resolution. It was noteworthy that standard deviation was extremely high with the maximum resolution, 3500-pixel.

The source of time consumption for classification, blob processing, and segmentation is specifically presented in Fig. 6(b). The blob

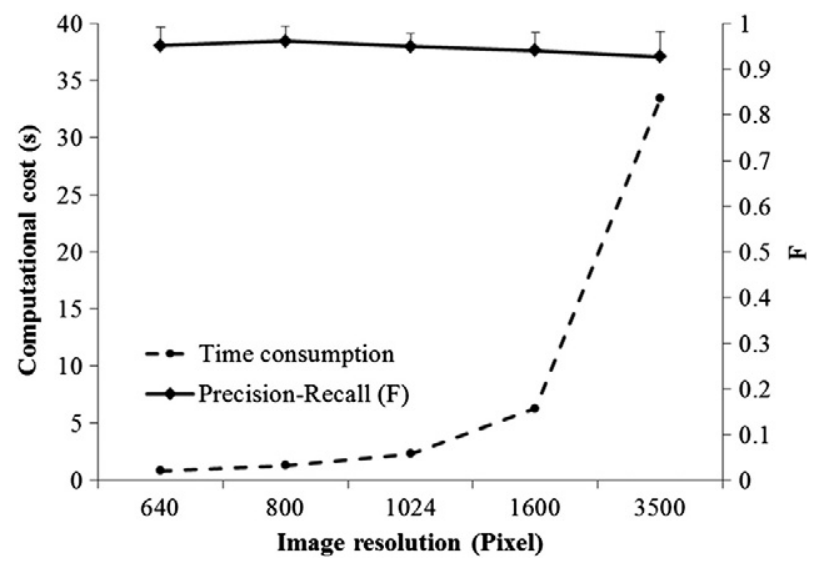

Fig. 5. Computational cost (s) and precision (F) across different levels of image resolution (Error bars indicate standard deviation of precision).
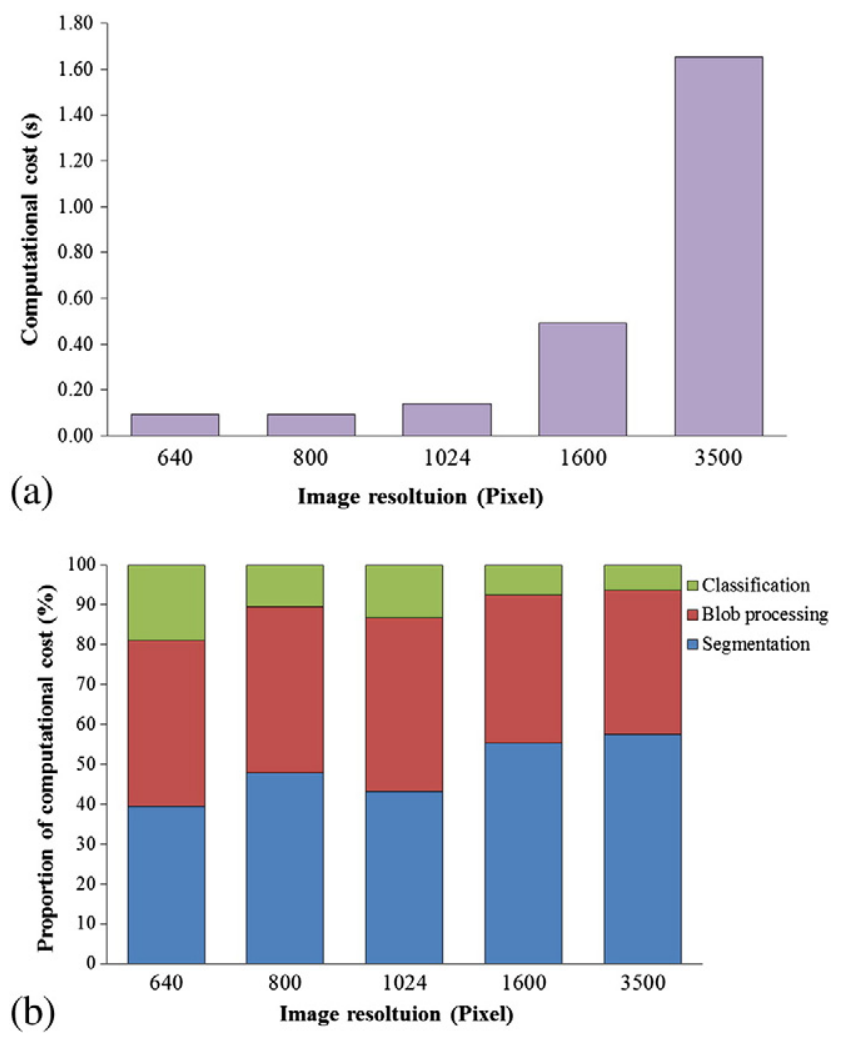

Fig. 6. Variation and computational cost (s). (a) Standard deviation, and (b) cost proportion (\%) of specific identification processes, including classification, blob processing and segmentation, in relation to the total time cost across different levels of image resolution.

processing includes morphological processing (e.g., erosion and dilation) and removal of the blob with unsuitable size (i.e., too large or too small sized blobs). Due to over-segmentation problems occurred with the high quality images, more blobs were produced by the segmentation processing. In addition to the image segmentation, blob processing on the high quality images cost much higher in dealing with the segmented blobs, comparing with the processing time with the low quality images. The computational time for both segmentation and blob processing dramatically increased by more than 20 times from 640-pixel images (1.06 s) to 3500-pixel images (34.51 s)(Fig. 5). This is understandable since the number of pixels increased 30 times from 640-pixel to 3500-pixel in resolution. The time for identification also increased substantially about 11 times at the same image resolution. On the contrary, the proportion of the classification time in relation to the total time cost decreased from $19.0 \%$ to $6.4 \%$ in images with maximal resolution (Fig. 6b). The computational cost of the Mahalanobis classifier was rather low, even the image resolution increased greatly. In fact, the increase of classification time was mainly caused by color feature extraction, since the insect image size geometrically increased with increase in pixels.

To evaluate pest identification accuracy the number of individuals for each species was separately counted by humans with experience in insect identification. It was noteworthy that the humans needed 5 to 20 minutes to examine the pests collected from one sticky trap. Maximal identification time was 40.6 seconds on the highest image resolution (3500-pixel). The average time consumptions by the automatic identification, however, were only 1.06 seconds on 640-pixel, 1.33 seconds for 800-pixel and 2.35 seconds for 1024-pixels.

The accuracy of species identification was evaluated by correlation of determination $\left(R^{2}\right)$ between the automatic identification and human counting (Table 1 ). The overall highest accuracy was achieved with whitefly maintaining maximal $R^{2}$ ranging $0.927-0.957$ across different image resolutions (Table 1 ). Aphid showed the maximum 
Table 1

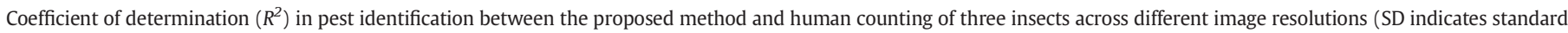
deviation).

\begin{tabular}{|c|c|c|c|c|c|c|}
\hline \multirow[t]{2}{*}{ Insect } & \multicolumn{5}{|c|}{ Image resolution (pixel) } & \multirow[t]{2}{*}{ Average \pm SD } \\
\hline & 640 & 800 & 1024 & 1600 & 3500 & \\
\hline Aphids & 0.881 & 0.934 & 0.941 & 0.912 & 0.909 & $0.915 \pm 0.023$ \\
\hline Thrips & 0.916 & 0.925 & 0.853 & 0.692 & 0.508 & $0.779 \pm 0.093$ \\
\hline Whitefly & 0.951 & 0.945 & 0.957 & 0.937 & 0.927 & $0.943 \pm 0.007$ \\
\hline Average \pm SD & $0.916 \pm 0.029$ & $0.935 \pm 0.008$ & $0.917 \pm 0.046$ & $0.847 \pm 0.110$ & $0.781 \pm 0.193$ & \\
\hline
\end{tabular}

recognition rate across different image resolutions, comparing with other species. The highest identification accuracy (0.941) was obtained from image resolution of 1024-pixel for aphid. The recognition rate of thrip was high with image resolutions of 640,800 and 1024 pixels, showing correlation of determination $\left(R^{2}\right)$ as $0.916,0.925$ and 0.853 , respectively. It was worth noting that the correlation of determination $\left(R^{2}\right)$ was only 0.692 and 0.508 with the high image quality (1600 and 3500 pixels images) for thrips. This was mainly due to false alarms. Identification accuracy of thrips considerably decreased when image resolution was higher than 800 pixels. The overall identification performance for three species was superior with image resolution of 800 pixels (0.935), followed by 1024 pixels (0.917) and 640 pixels (0.916) (Table 1).

Precision analysis (Table 2) was further performed to examine the correctness achieved by identification results; a high precision value indicates that the identification results contain a high percentage of correctly identified pests and a low percentage of false alarms as well (Xia et al., 2012), as stated above. False alarms frequently occurred in the identification results, affecting overall identification accuracy. The precision analysis reveals the reliability of the identification results. As shown in Table 2, the precision values are comparatively higher in the low image resolutions. Precision was 0.905 for aphid, 0.955 for thrip and 0.871 for whitefly with image resolution of 640 pixels whereas the values were $0.941,0.869$ and 0.870 with 800 pixels, respectively. The precision was in general lower in the higher resolution of images ( $>1024$ pixels). The precision of identifying thrips and whitefly even decreased significantly to 0.692 and 0.654 , respectively, with maximum image resolution of 3500 pixels (Table 2). Since over-segmentation increased in high resolution images, false alarms correspondingly increased to induce reduction in precision. Inaccurate segmentation, which often contained the surrounded background in the insect images, also caused the inaccurate mean color of insects for identifying pest species. Aphids overall showed the best recognition performance $\left(R^{2}\right)$ (Table 2) with different image resolutions although the maximum precision was achieved with whitefly (Table 1 ). Due to distinctive size (i.e., large) and color (i.e., black), aphids overall showed higher feasibility in identification comparing with other species.

The confusion matrix presents the degree of correct answer along with the identification errors for the three insects (Fig. 7) (Kohavi and Provost, 1998). The diagonal elements of the confusion matrix indicate the true positives. The rest of elements in rows represent the false positives of classification. Overall diagonal elements showed the maximum values as expected. Substantially small numbers were found at either row or column elements. The values decreased in general along with increase in image resolution, especially at maximum resolution for whitefly and thrips. For instance the thrips were confused with whitefly, and vice versa, at maximum resolution, showing the error rates as 0.144 and 0.087 , respectively, whereas the values were substantially lower at other resolutions (Fig. 7). Whitefly and thrips tended to be easily confused in the classification results. Caution is needed in identifying aphids. Segmentation of aphids occasionally ended up with several small segments, some of which were recognized as single individual although the segment is only a part of the body.

By taking into account the correlation of determination, precision analysis, and the computational cost all together (Tables 1 and 2, and Fig. 7), the image resolution of 800 -pixel could be an optimal choice for detecting and counting the three major pests in greenhouse. The image resolution of 640-pixel could be also an option to detect pests in practical aspect, considering low computational cost.

\section{Discussion}

Cho et al. (2007) reported the segmentation of three species, aphids, whitefly and thrips, by two different approaches: aphids were detected in RGB image whereas whitefly and thrips were segmented in YUV color space. In this study, three species of pests were segmented based on one method for concurrent identification of three species from the sticky trap images. The preliminary experiments of insect segmentation were conducted on widely used color spaces including CIELab, HSV and $\mathrm{YC}_{\mathrm{b}} \mathrm{C}_{\mathrm{r}}$ color models (Payne et al., 2014; Sural et al., 2002; Zheng and $\mathrm{Lu}, 2012$ ). The $\mathrm{YC}_{\mathrm{b}} \mathrm{C}_{\mathrm{r}}$ color model showed the better segmentation comparing with CIELab and HSV. In our study the insects and grids on the sticky traps were presented as local maxima in the $C_{b}$ channel of $\mathrm{YC}_{\mathrm{b}} \mathrm{C}_{\mathrm{r}}$ color model (Fig. 2c). Consequently the local maxima on the image could be extracted as the markers for the marker-controlled watershed segmentation. The green grids, however, showed much lower intensity values in the $C_{b}$ channel than common greenhouse species (Fig. 2c). Therefore, the accuracy of insect segmentation was higher in the $C_{b}$ channel. The segmentation from the yellow sticky traps was also demonstrated with the green color insects such as greenfly (Kumar et al., 2010), however the segmentation in this case was only conducted with the traps without printed green grids and alphabets. We demonstrated that segmentation was even possible with images showing grids and alphabets in this study (Fig. 2a).

Although multifractal demonstrated showed higher performance regarding accuracy than watershed on insect segmentation against noise in field conditions (e.g., illumination reflections) (Xia et al., 2012), multifractal is much more computationally expensive. Consequently, automation with multifractal would not be affordable for long term operation of portable devices. Otsu algorithm (Otsu, 1979) demands less

Table 2

Precision in pest identification of three insects across different image resolutions (SD indicates standard deviation).

\begin{tabular}{|c|c|c|c|c|c|c|}
\hline \multirow[t]{2}{*}{ Insects } & \multicolumn{5}{|c|}{ Image resolution (pixel) } & \multirow[t]{2}{*}{ Average \pm SD } \\
\hline & 640 & 800 & 1024 & 1600 & 3500 & \\
\hline Aphids & 0.905 & 0.941 & 0.858 & 0.828 & 0.746 & $0.856 \pm 0.043$ \\
\hline Thrips & 0.955 & 0.869 & 0.790 & 0.806 & 0.692 & $0.822 \pm 0.065$ \\
\hline Whitefly & 0.871 & 0.870 & 0.818 & 0.773 & 0.654 & $0.797 \pm 0.041$ \\
\hline Average \pm SD & $0.910 \pm 0.034$ & $0.893 \pm 0.034$ & $0.822 \pm 0.028$ & $0.802 \pm 0.023$ & $0.697 \pm 0.038$ & \\
\hline
\end{tabular}




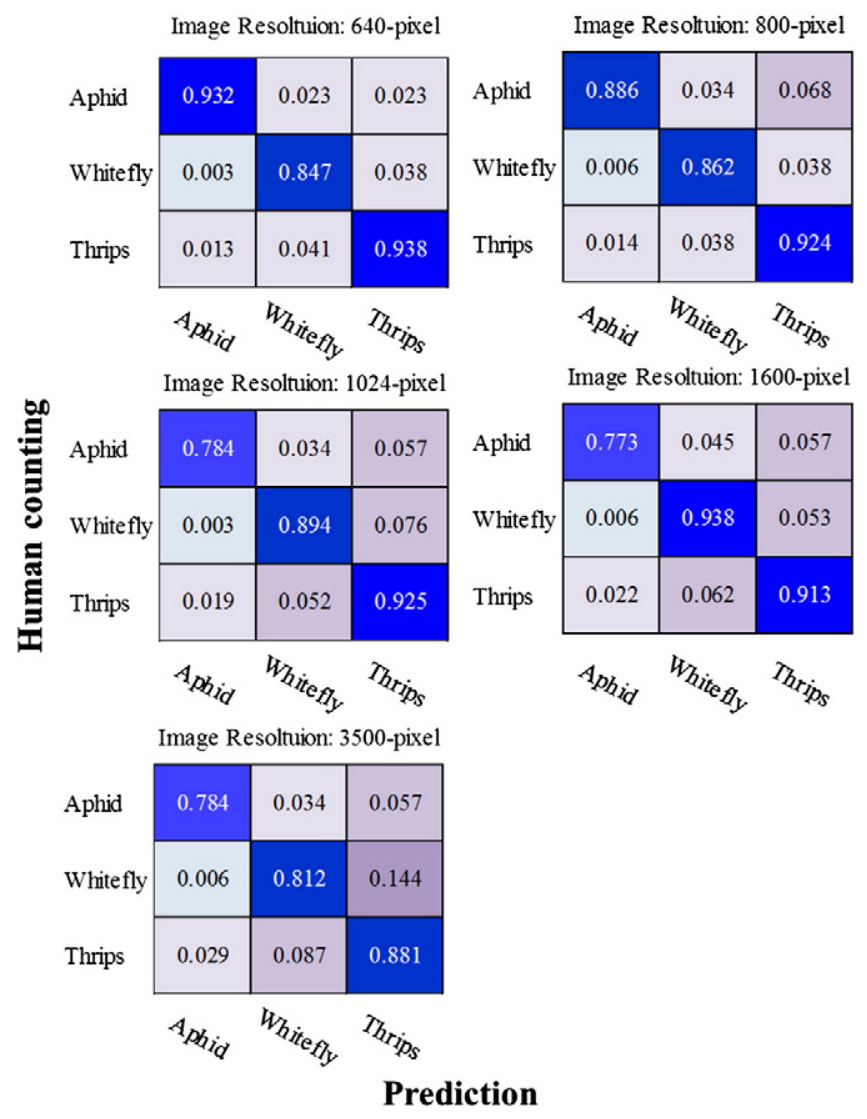

Fig. 7. Confusion matrices for human counting and predication in recognition of three insects across different image resolutions (Darker color indicates higher accuracy for diagonal elements).

computational cost, however the method is mainly designed for global thresholding and is highly sensitive to illumination changes (Xia et al., 2012). The local adaptive thresholding (Kom et al., 2007) could be another option to extract the insects from sticky trap images without green grids as reported by Kumar et al. (2010) and Bechar et al. (2010). In this case, however, the green grids on the sticky traps should be separately segmented before identifying insects. Considering extraction of the green grids, the watershed (Vincent and Soille, 1991) could be an optimal solution to acquire accurate segmentation of insects from backgrounds in acceptable precision level while achieving lower computational cost concurrently. Computational complexity of insect segmentation could be further reduced by the integrating adaptive thresholding algorithms (Rahman and Islam, 2013). This will be investigated in the future study.

Segmentation accuracy showed a slightly decreasing trend as resolution increased in this study (Fig. 5). This was due to hyper sensitivity to noise caused by high image resolution. Although high quality images (>1600 pixels) contained details of the pests, the detection results turned out to be too sensitive to environmental effects (e.g., illumination change). Details of image were amplified in high resolution and more local maxima were produced while extracting pest images from backgrounds. Since the watershed segmentation was based on the local maxima, the large number of local maxima led to production of more watershed segmentations, causing hypersensitivity to noise subsequently. The inaccurate segmentation or partially segmented insect images were an important source of the identification errors according to authors' experience. Legs and wings of the large aphids, for example, were occasionally segmented as separate objects to serve as extra noise sources. The ill-segmented objects were sometimes incorrectly identified as different species (e.g., thrips) to make a false signal (Fig. 8a). Since thrips' body contains gray and white areas together,

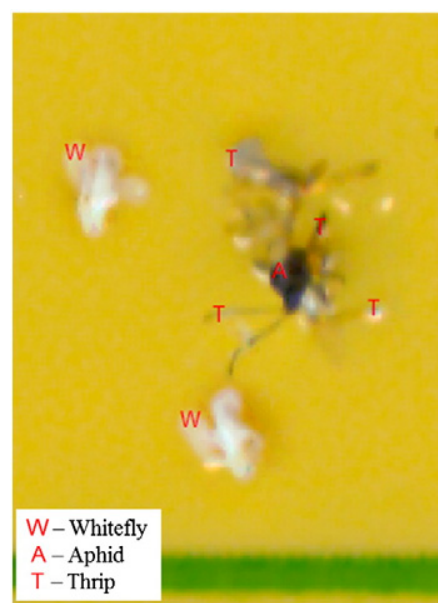

(a)

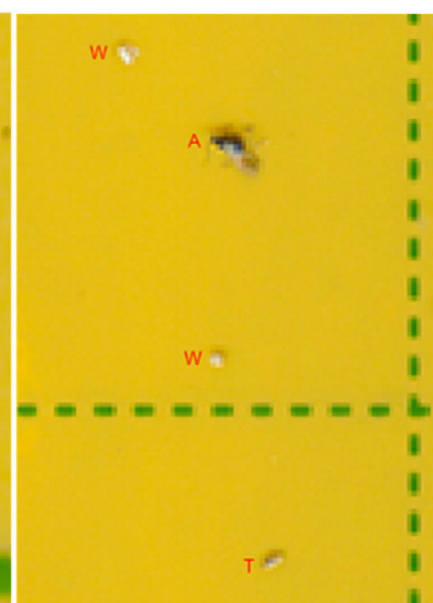

(b)
Fig. 8. Images of small size pests difficult for recognition. (a) Over-segmentation of large pests, and (b) ambiguous objects for identification.

thrips were occasionally divided into two parts in the high resolution images (Tables 1 and 2). With low resolution images on the other hand, image feature of legs and wings were not strong and were neglected by the segmentation algorithm. The optimal level of resolution was required as shown in this study.

In order to deal with the high quality images, more sophisticated algorithms would be necessary such as scale invariant feature transformation (SIFT) (Solis-Sánchez et al., 2011). However, since the appearance of three species (thrips, aphids and whitefly) were mainly distinguishable with color features in field conditions, the complicated algorithms applied to high quality images were not necessary to detect and identify pests collected on sticky traps in field conditions in practical aspect as demonstrated in this study.

Although aphids showed the best recognition performance, inaccurately segmented aphids led to incorrect identifications. Some segmented images of aphids contained their legs or the surrounding backgrounds, therefore, the mean color of the aphid was sometimes distorted since the legs and background color were included in calculating the mean color. The aphids could be miss-recognized as thrips in these cases. The incorrect identification of aphids, however, was not frequent, especially with the low image resolution.

Identification performance of thrips was relatively lower comparing with other species, since small size thrips are often confused with whitefly as presented in the confusion matrices (Fig. 7). As presented in Fig. 8(b), the pest in the center was identified as whitefly and marked with a red letter ' $W$ '. The mean color of that pest was close to whitefly but the pest body was in fact surrounded by gray shadow, which also fit to the feature of thrips. These pests were incorrectly identified as whitefly in this case as shown in Fig. 8(b). In addition, the overlapped image of specimens may also reduce the identification accuracy. The pest size was rather smaller in this study, and there was a low possibility of overlapping. In the future, however, new method should be devised in case pest population size increased and overlapping of specimens would increase on the sticky trap.

Light condition was an additional problem for insect identification. Illumination conditions vary in a high degree in natural condition and would cause color distortion in image capturing in greenhouse especially with high resolution images. In this study, it was aimed to present a prototype of automatic pest identification system, and the current version of the proposed method still needs further implementation tests in response to various environmental factors (e.g., continuously changing illumination conditions) and different population size to build the products for satisfactory use in agricultural practices. Color correction (Rizzi et al., 2003) or automatic parameter tuning 
(Boissard et al., 2008) should be also considered in this regard in the future. In addition, real scale field tests would be further needed to evaluate identification performance over space (e.g., whole green house) and time (e.g., during whole procedure of cultivation). Further study is required in this regard with the aid of field assessment methods and integrative pest management programs.

\section{Conclusion}

By using watershed algorithm and Mahalanobis distance on $\mathrm{YC}_{\mathrm{r}} \mathrm{C}_{\mathrm{b}}$ color space, a pest identification procedure was proposed, achieving both low computational cost and high feasibility in identifying three common species, aphid, thrips and whitefly, in greenhouse. The pest detection and species identification accuracy were verified through experiments with specimens collected on sticky traps in greenhouse conditions. The proposed method demonstrated high performance in identifying and counting small sized pests especially with low resolution images (i.e., 800-pixel, 640-pixel) when compared with human identification results. The proposed method would be especially practical for large scale, long term monitoring by using the portable devices which require minimal power consumption and endurable data storage.

\section{References}

Allen, W.A., Rajotte, E.G., 1990. The changing role of extension entomology in the IPM era. Annu. Rev. Entomol. 35 (1), 379-397.

Bechar, I., Moisan, S., Thonnat, M., Bremond, F., 2010. On-line video recognition and counting of harmful insects. The 20th International Conference on Pattern Recognition (ICPR), pp. 4068-4071.

Boissard, P., Martin, V., Moisan, S., 2008. A cognitive vision approach to early pest detection in greenhouse crops. Comput. Electron. Agric. 62 (2), 81-93.

Bommarco, R., Kleijn, D., Potts, S.G., 2013. Ecological intensification: harnessing ecosystem services for food security. Trends Ecol. Evol. 28 (4), 230-238.

Cho, J., Choi, J., Qiao, M., Ji, C.W., Kim, H.Y., Uhm, K.B., Chon, T.S., 2007. Automatic identification of whiteflies, aphids and thrips in greenhouse based on image analysis. Int. J. Math. Comput. Simul. 346 (246), 244.

Chung, B.K., Xia, C., Song, Y.H., Lee, J.M., Li, Y., Kim, H., Chon, T.S., 2014. Sampling of Bemisia tabaci adults using a pre-programmed autonomous pest control robot. J. Asia Pac. Entomol. 17 (4), 737-743.

De Maesschalck, R., Jouan-Rimbaud, D., Massart, D.L., 2000. The mahalanobis distance. Chemom. Intell. Lab. Syst. 50 (1), 1-18.

Gaston, K.J., O'Neill, M.A., 2004. Automated species identification: why not? Philos. Trans. R. Soc. Lond. B Biol. Sci. 359 (1444), 655-667.

Kato, N., Suzuki, M., Omachi, S.I., Aso, H., Nemoto, Y., 1999. A handwritten character recognition system using directional element feature and asymmetric Mahalanobis distance. IEEE Trans. Pattern Anal. Mach. Intell. 21 (3), 258-262.

Kohavi, R., Provost, F., 1998. Glossary of terms: special issue on applications of machine learning and the knowledge discovery process. Mach. Learn. 30, 271-274.

Kom, G., Tiedeu, A., Kom, M., 2007. Automated detection of masses in mammograms by local adaptive thresholding. Comput. Biol. Med. 37 (1), 37-48.

Koumpouros, Y., Mahaman, B.D., Maliappis, M., Passam, H.C., Sideridis, A.B., Zorkadis, V. 2004. Image processing for distance diagnosis in pest management. Comput. Electron. Agric. 44 (2), 121-131.
Kumar, R., Martin, V., Moisan, S., 2010. Robust insect classification applied to real time greenhouse infestation monitoring. Proceedings of the 20th International Conference on Pattern Recognition on Visual Observation and Analysis of Animal and Insect Behavior Workshop, pp. 1-4.

MacLeod, N., Benfield, M., Culverhouse, P., 2010. Time to automate identification. Nature 467 (7312), 154-155.

Malais, M.H., Ravensberg, W.J., 2004. Knowing and Recognizing: The Biology of Glasshouse Pests and Their Natural Enemies. Koppert BV, The Netherlands.

Manh, A.G., Rabatel, G., Assemat, L., Aldon, M.J., 2001. AE-automation and emerging technologies: weed leaf image segmentation by deformable templates. J. Agric. Eng. Res. 80 (2), 139-146.

Martin, V., Thonnat, M., 2007. A cognitive vision approach to image segmentation. Proceedings of the 19th IEEE International Conference on Tools with Artificial Intelligence Washington, DC, USA, pp. 265-294.

Ng, H.P. Ong S.H. Foong K.W.C. Goh, P.S, Nowinski, W.L, 2006. Medical image segmentation using K-means clustering and improved watershed algorithm. IEEE Southwest Symposium on Image Analysis and Interpretation, pp. 61-65.

Otsu, N., 1979. A threshold selection method from gray-level histograms. Automatica 11 (285-296), 23-27.

Parvati, K., Rao, P., Mariya Das, M., 2008. Image segmentation using gray-scale morphology and marker-controlled watershed transformation. Discret. Dyn. Nat. Soc. 8 (Article ID 384346).

Payne, A., Walsh, K., Subedi, P., Jarvis, D., 2014. Estimating mango crop yield using image analysis using fruit at 'stone hardening' stage and night time imaging. Comput. Electron. Agric. 100, 160-167.

Pinto-Zevallos, D.M., Vänninen, I., 2013. Yellow sticky traps for decision-making in whitefly management: what has been achieved? Crop. Prot. 47, 74-84.

Qiao, M., Lim, J., Ji, C.W., Chung, B.K., Kim, H.Y., Uhm, K.B., Myung, C.S., Cho, J., Chon, T.S. 2008. Density estimation of Bemisia tabaci (Hemiptera: Aleyrodidae) in a greenhouse using sticky traps in conjunction with an image processing system. J. Asia Pac. Entomol. 11 (1), 25-29.

Rahman, M., Islam, M., 2013. Segmentation of color image using adaptive thresholding and masking with watershed algorithm. Proceedings of International Conference on Informatics, Electronics \& Vision, pp. 1-6.

Rizzi, A., Gatta, C., Marini, D., 2003. A new algorithm for unsupervised global and loca color correction. Pattern Recogn. Lett. 24 (11), 1663-1677.

Solis Sánchez, L., García Escalante, J., Castañeda Miranda, R., Torres Pacheco, I., Guevara González, R., 2009. Machine vision algorithm for whiteflies (Bemisia tabaci Genn.) scouting under greenhouse environment. J. Appl. Entomol. 133 (7), 546-552.

Solis-Sánchez, L.O., Castañeda-Miranda, R., García-Escalante, J.J., Torres-Pacheco, I., Guevara-González, R.G., Castañeda-Miranda, C.L., Alaniz-Lumbreras, P.D., 2011 Scale invariant feature approach for insect monitoring. Comput. Electron. Agric. 75 (1), 92-99.

Sural, S., Qian, G., Pramanik, S., 2002. Segmentation and histogram generation using the HSV color space for image retrieval. Proceedings of International Conference on Image Processing 2, p. II-589.

Vincent, L., Soille, P., 1991. Watersheds in digital spaces: an efficient algorithm based on immersion simulations. IEEE Trans. Pattern Anal. Mach. Intell. 13 (6), 583-598.

Weinberger, K., Blitzer, J., Saul, L., 2006. Distance metric learning for large margin nearest neighbor classification. Adv. Neural Inf. Process. Syst. 18, 1473

Wise, J.C., Gut, L.J., Isaacs, R., 2007. Michigan Fruit Management Guide. Department of Plant Pathology and Department of Horticulture, Michigan State University.

Xia, C., Lee, J.M., Li, Y., Chung, B.K., Chon, T.S., 2012. In situ detection of small-size insect pests sampled on traps using multifractal analysis. Opt. Eng. 51 (2), 027001-1.

Xia, C., Lee, J.M., Li, Y., Song, Y.H., Chung, B.K., Chon, T.S., 2013. Plant leaf detection using modified active shape models. Biosyst. Eng. 116 (1), 23-35.

Zheng, H., Lu, H., 2012. A least-squares support vector machine (LS-SVM) based on fractal analysis and CIELab parameters for the detection of browning degree on mango (Mangifera indica L.). Comput. Electron. Agric. 83, 47-51. 